\title{
Platelet-Leukocyte Complex Formation in Myocardial Infarction: a Marker not a Pathogen
}

$\underline{\text { C. Starz }}^{1}$, C. Härdtner ${ }^{1}$, M. Mauler², B. Dufner ${ }^{1}$, H. Natalie', D. Duerschmied ${ }^{1}$, C. Bode ${ }^{1}$, A. Zirlik ${ }^{3}$, I. Hilgendorf ${ }^{1}$.

UNIVERSITA'TS

HERZZENTRUM

1 University of Freiburg, Department of Cardiology and Angiology I - University Heart Center Freiburg-Bad Krozingen, Germany ${ }^{2}$ University of Munich, Department of Cardiovascular Prevention, Munich, Germany. ${ }^{3}$ University of Graz, Department of Cardiology, Graz, Austria.

\section{Immunocardiology}

\section{BACKGROUND}

Activated platelets and leukocytes form complexes via several adhesion molecules, most notably P-selectin and PSGL1 ${ }^{1}$. Increased numbers of platelet-leukocyte complexes (PLC) circulate in patients with acute myocardial infarction ${ }^{2}$ and predict adverse outcomes. It has therefore been proposed that PLC directly engage in adverse cardiac remodeling and progression of coronary artery disease. PLC formation is thought to promote adhesion of leukocytes to the vessel wall ${ }^{3,4}$ and infiltration into sites of inflammation ${ }^{5}$. In this work we used a platelet specific Pselectin-knockout mouse model to attenuate PLC formation without affecting endothelial or leukocyte bound adhesion molecules. To our surprise, leukocyte rolling and adhesion to the endothelium and infiltration into the inflamed peritoneum remained unaffected by the lack of PLC in knockout mice. We also detected no differences in neutrophil migration after ischemia-reperfusion injury in infarcted area. Our work challenges the prevailing paradigm that PLC are instrumental in supporting leukocyte invasion in tosites of steril inflammation in vivo ${ }^{6}$
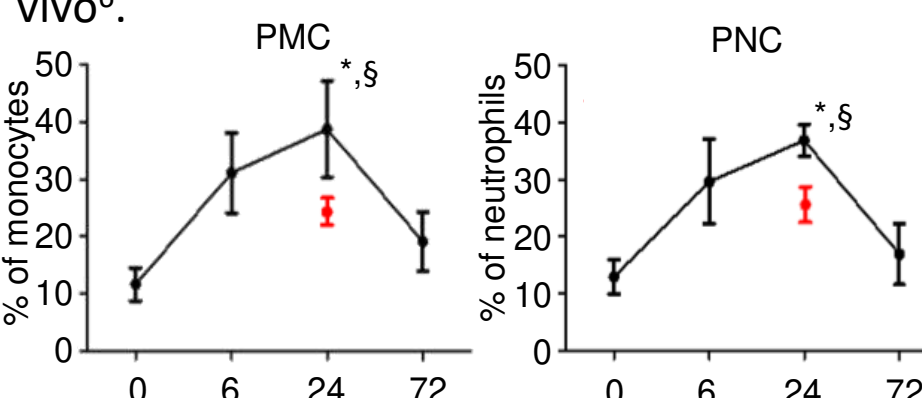

Platelet-monocyte (PMC) and -neutrophile (PNC) complexes in wild type mice (WT) at baseline (Oh), 6, 24 and $72 \mathrm{~h}$ of reperfusion after $35 \mathrm{~min}$ of ischemia by LAD Ligation measured by flow cytometry analysis. ). Results are presented as mean \pm SEM ${ }^{*} p<0.05$ Oh vs. $24 h ; \S p<0.0524 h$ vs. sham operated mice (red). $n=4 /$ time point

Yang 1999; ${ }^{2}$ Michelson 2001; ${ }^{3}$ Henderson et al 2001; ${ }^{4}$ Phillipson 2011; 5 Yang et al. 1999; ${ }^{6}$ Gaetano et al. 2003
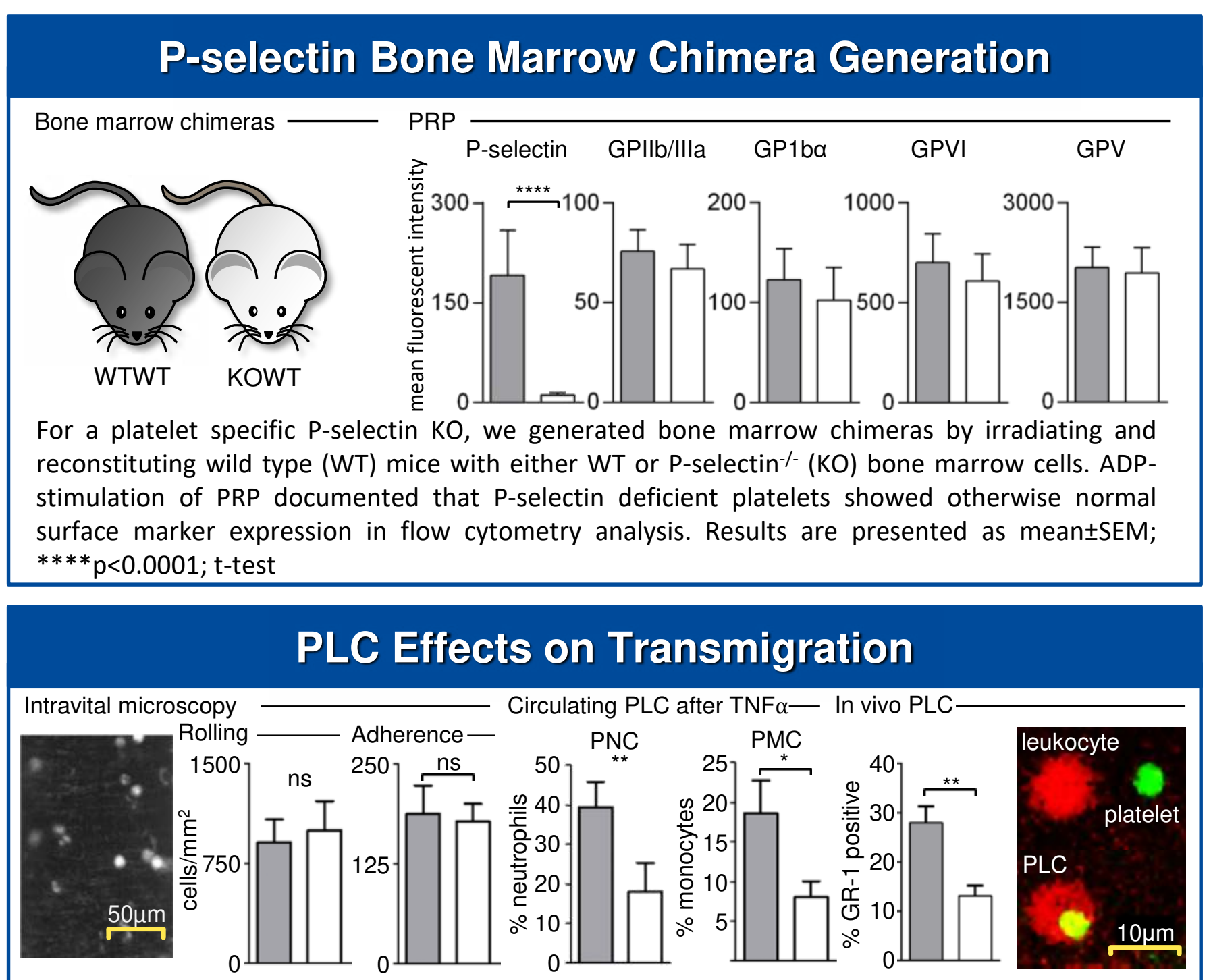

Steril peritonitis
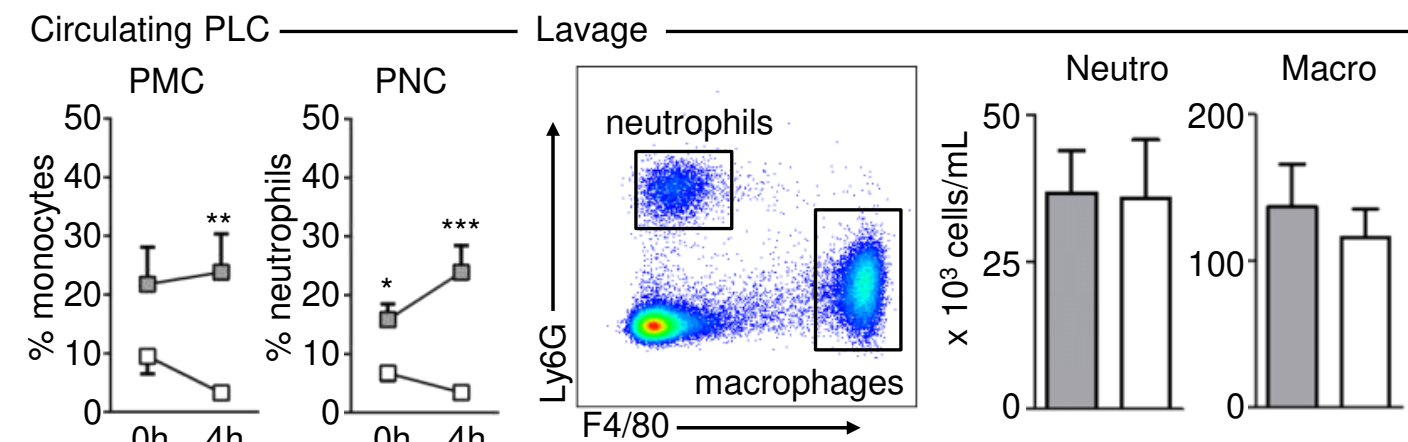

口 WTWT

Representative image of leukocytes rolling on TNF $\alpha$ activated endothelium. The lack of platelet derived P-selectin does not alter leukocyte rolling and adherence to activated endothelial cells in vivo, in intravital microscopy (top left). PLC levels remained significantly reduced in KOWT chimeras. We also confirmed significantly lower PLC levels in KOWT chimeras in vivo by two color intravital microscopy (top right). Intraperitoneal thioglycollate injection increases circulating PLC levels only in WTWT chimeras after $4 \mathrm{~h}$ incubation (bottom left). Representative flow cytometry gating strategy for neutrophils and macrophages from peritoneal lavage. Despite significantly lower PLC levels in KOWT chimeras we observed no differences in neutrophil or macrophage cell counts in peritoneal lavage. (ns=not significant; ${ }^{*} \mathrm{p}<0.05 ;{ }^{* *} \mathrm{p}<0.01 ;{ }^{* * *} \mathrm{p}<0.001$ ).

Immunocardiology Research Group - AG Hilgendorf

Univerity Heart Center Freiburg - Bad Krozingen - Breisacherstraße 33 - 79106 Freiburg - Germany

E-mail: ingo.hilgendorf@universitaets-herzzentrum.de

Phone: +49(0)761/270-70081

\section{The Role of PLCs in Ischemia Reperfusion Injury}
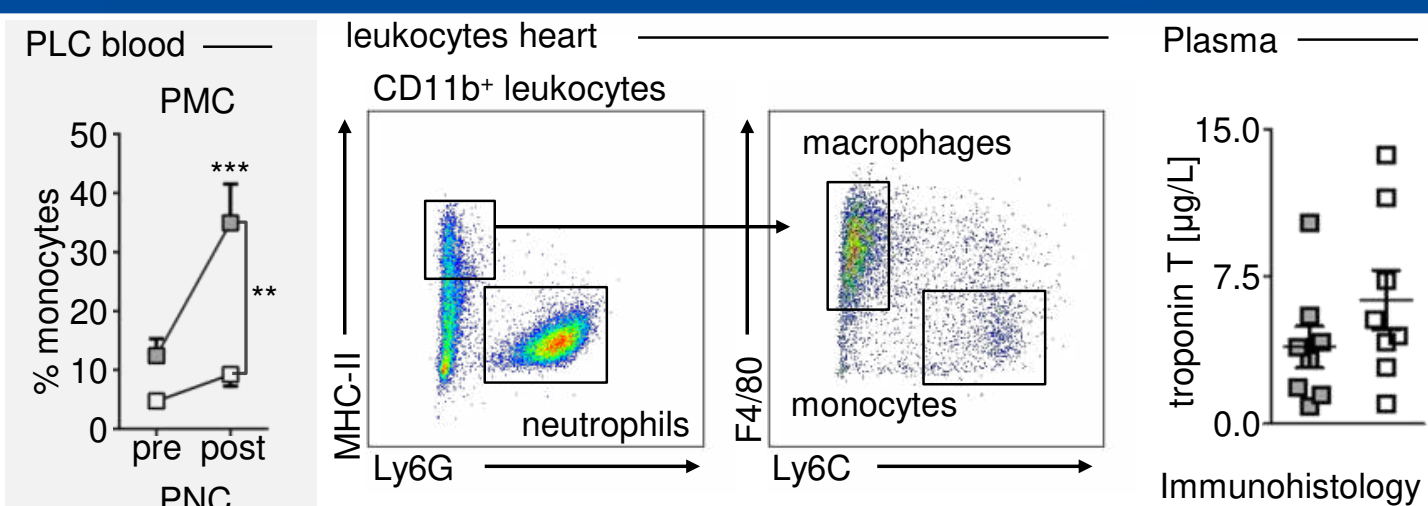

ㅁ WTWT
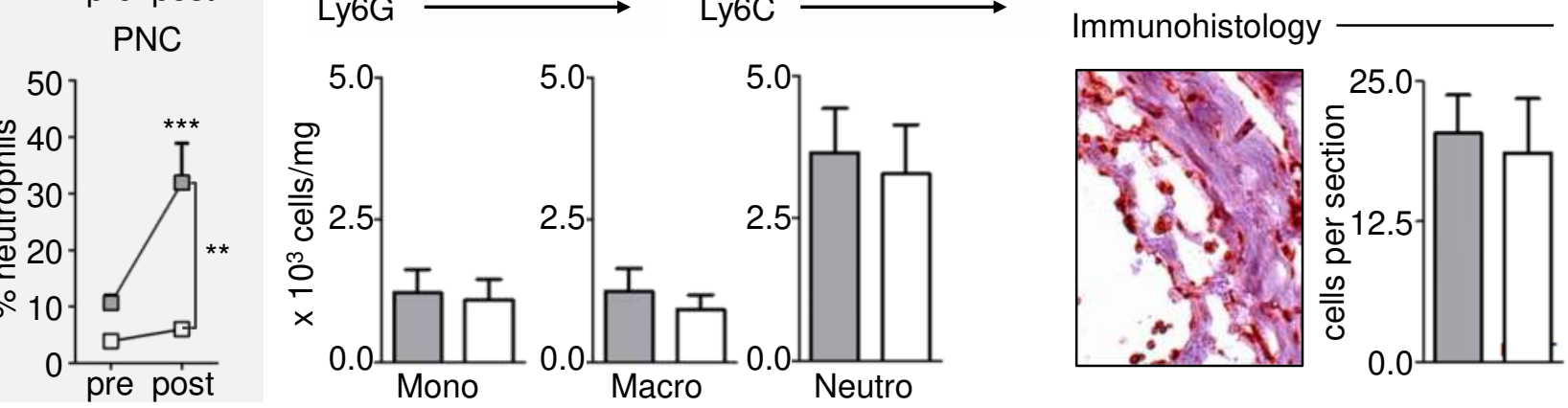

PLC levels in circulation before (pre) and $24 \mathrm{~h}$ after (post) 35 min of LAD ligation. There was no PLC increasement in KOWT chimeras (left). Gating strategy for leukocytes in lysated infarcted myocardium (top middle). No differences in monocyte, macrophage or neutrophil counts in infarcted myocardium (bottom middle). Plasma troponin T levels showed no differences between WTWT and KOWT chimeras (top left). We also found no differences in Ly6G positiv cellcounts in infarcted myocardium after $24 \mathrm{~h}$ LAD ligation by immunohistology. (Results are presented as mean \pm SEM; ${ }^{* *} \mathrm{p}<0.01 ; * * * \mathrm{p}<0.001 ;$ t-test $)$.

\section{PLC Impact on Infarct size and Long Term Effects}
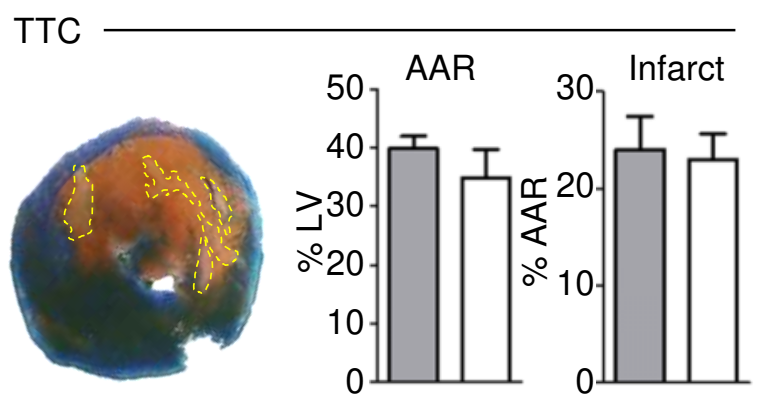

The comparison of area at risk (AAR, red) and actual infarct size (yellow dashed) between both groups showed no differences in TTC staining (top left) Longterm functional analysis by echocardiography at baseline (d0) and 21 days after 35 min of LAD ligation showed in WTWT and KOWT an equal decrease of left ventricular ejection fraction (LVEF) and fractional shortening (FS). Both groups also showed an uniform increase in left ventricular endsystolic and enddiastolic diameters (LVESD and LVEDD, right).

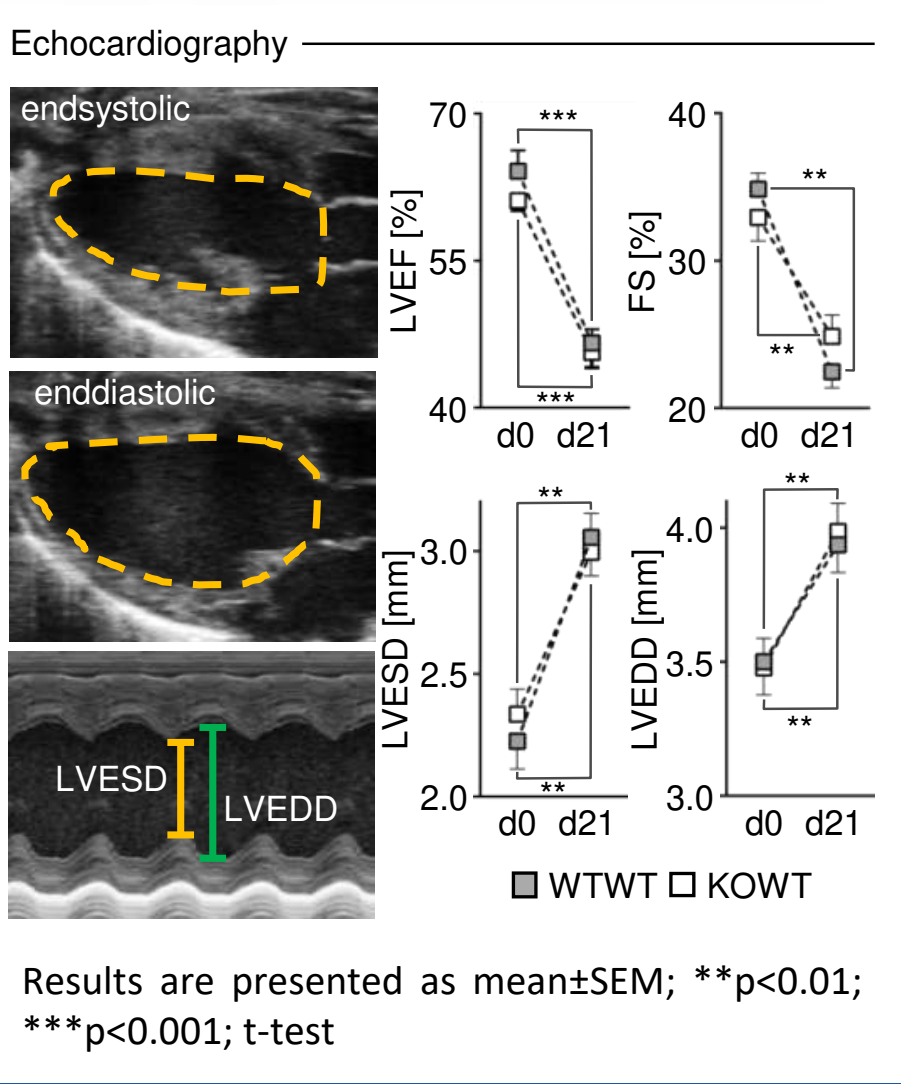

PLC formation may promote bacterial digestion

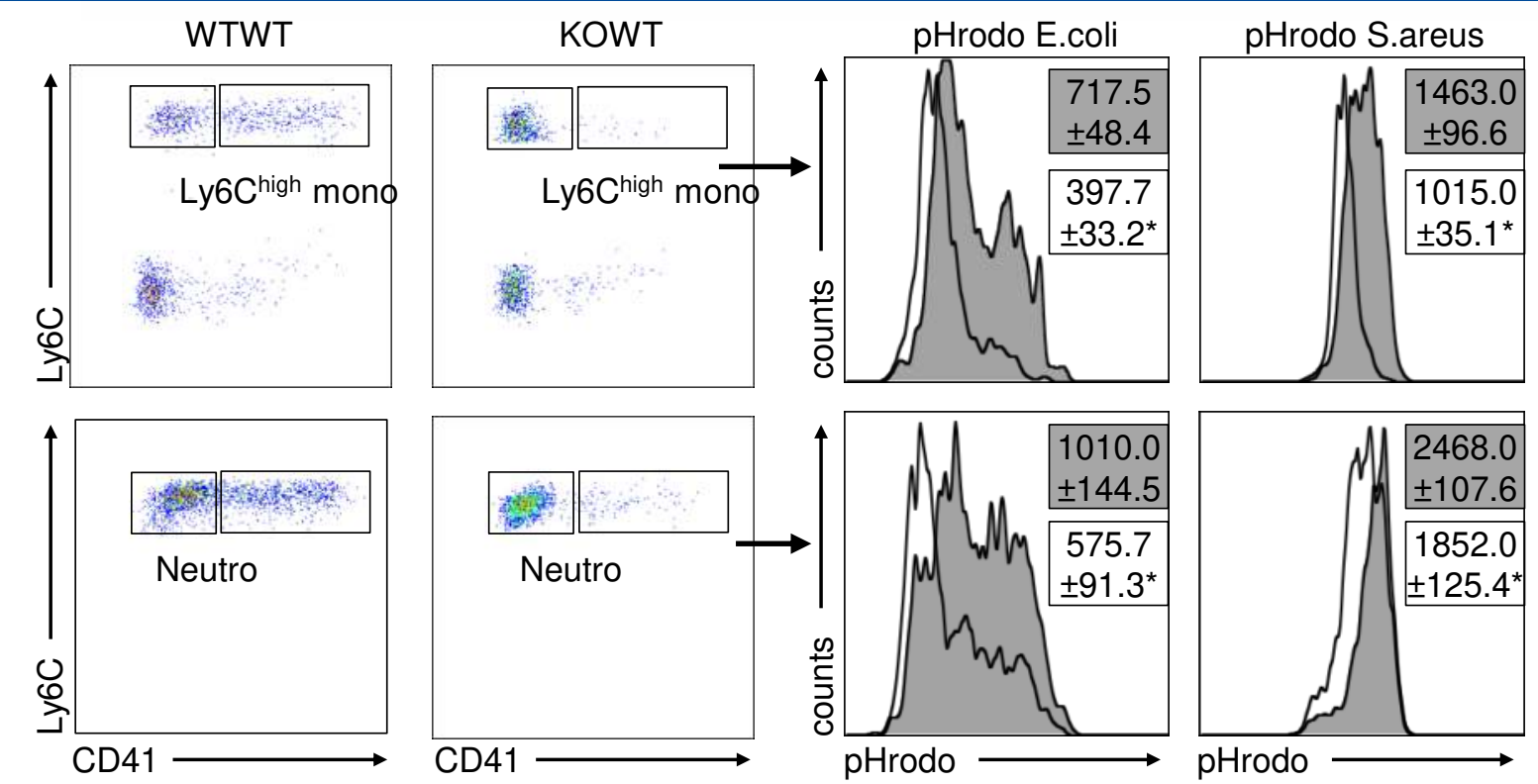

Representative flow cytometry gating strategy for Ly6 $6 \mathrm{C}^{\text {high }}$ monocytes and neutrophils (left). Flow cytometric analysis of pHrodo digestion. KOWT mice with significant reduced PLC formation show less pHrodo E.coli and less pHrodo $\mathrm{S}$. aureus particle digestion measuerd by mean flurescence intensity. Results are presented as mean $\pm S E M ;{ }^{*} p<0.05$; t-test

\section{ConcJusion}

- $\quad$ platelet derived P-selectin is dispensible for leukocyte invasion to sites of steril inflammation

circulating PLC have no influence on rolling, adherence or transmigration of leukocytes in postcapillary venules

- $\quad$ PLC are rather a marker than a pathogen in myocardial infarction

- Circulating PLC may promote bacterial phagocytosis 